\title{
Capacitor Cilíndrico Excêntrico
}

\author{
Eccentric cylindrical capacitor \\ Ricardo Egydio de Carvalho e Ana Paula Morinelli Amaral da Silva \\ Departamento de Estatística, Matemática Aplicada e Computação, \\ Instituto de Geociências e Ciências Exatas, Universidade Estadual Paulista \\ C.P. 178, 13500-230, Rio Claro, SP, Brasil \\ E-mail dos autores: regydio@rc.unesp.br,apmas@rc.unesp.br
}

Recebido em 7 de maio, 2002. Aceito em 7 de junho, 2002.

\begin{abstract}
Consideramos o capacitor cilíndrico com a placa interna fora de centro e calculamos a capacitância correspondente usando a técnica da transformação conforme. Observamos que a capacitância pode ser ampliada e a quantidade de material para construir o capacitor pode ser reduzida com a geometria excêntrica.Este resultado pode ser usado como tutorial de uma aula experimental para medir o efeito da excentricidade na capacitância e na construção do capacitor cilíndrico.
\end{abstract}

\begin{abstract}
We consider the cylindrical capacitor with the inner plate out of centre and we calculate the corresponding capacitance using the conformal transformation technique. We observe that the capacitance may be amplified and the quantity of material to construct the capacitor may be reduced with the eccentric geometry. This result can be used as a tutorial for an experimental lecture in order to measure the effect of the eccentricity in the capacitance and also in the construction of the cylindrical capacitor.
\end{abstract}

\section{Capacitores}

\section{I.1 A utilização dos capacitores}

Um capacitor é um dispositivo utilizado para armazenar energia, na forma de energia potencial, contida em campos elétricos. Os capacitores têm várias aplicações além de servirem como armazenadores de energia. Eles constituem elementos importantes nos circuitos elétricos de transmissores e de receptores de rádio e televisão. Os capacitores microscópicos formam os bancos de memória dos computadores. Tais campos elétricos são significativos também pela informação ligadesliga que a presença ou ausência deles proporciona.

\section{I.2 Capacitância}

Os capacitores se apresentam numa grande variedade de tamanhos e formas. Entretanto, os elementos básicos de qualquer capacitor são dois condutores isolados de formatos arbitrários. Chamamos tais condutores de placas, quaisquer que sejam suas geometrias. São bem conhecidos os capacitores de placas paralelas, os esféricos e os cilíndricos.

Quando um capacitor é carregado, suas placas adquirem cargas iguais, mas de sinais opostos, $+Q$ e $-Q$. Entretanto, referimo-nos à carga do capacitor como sendo meramente $Q$, o valor absoluto das cargas sobre as placas.
Uma vez que as placas são condutoras, elas constituem superfícies equipotenciais. Além disso, existe uma diferença de potencial entre as duas placas, representada por $V_{1}$.

A carga $Q$ e a diferença de potencial $V_{1}$ para um capacitor são proporcionais entre si e mediadas por uma grandeza $C$, isto é,

$$
Q=C V_{1}
$$

onde a constante de proporcionalidade $C$ é chamada capacitância do capacitor. Este parâmetro, tal como é conhecido, deve depender apenas da geometria das placas. No entanto, mostraremos um resultado interessante neste trabalho.

A unidade da capacitância no Sistema Internacional de unidades é o coulomb por volt, ou farad $(\mathrm{F})$.

\section{I.3 O Capacitor Cilíndrico}

A Fig. 1 mostra a seção transversal de um capacitor cilíndrico de comprimento $L$, formado por dois cilíndricos coaxiais de raios $R_{1}$ e $R_{2}$. Supomos que $L>R_{1}$, de modo que podemos desprezar a "distorção" do campo elétrico que ocorre nas extremidades dos cilindros. Cada placa contém uma carga de módulo $Q$. O potencial elétrico na placa externa é igual à $V_{1}$, enquanto a placa interna encontra-se aterrada, isto é, $V=0$. 


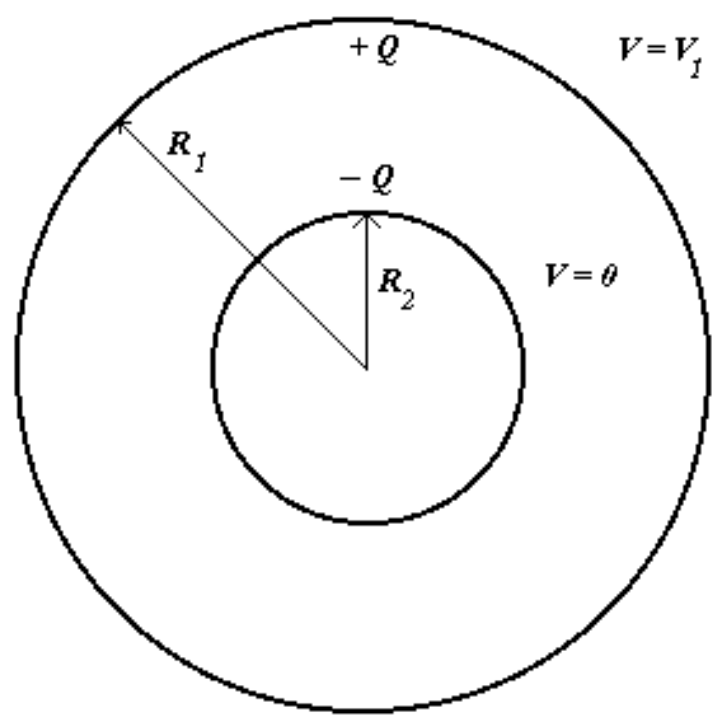

Figura 1. Seção transversal de um capacitor cilíndrico com raios $R_{1}$ e $R_{2}$. A placa interna encontra-se aterrada enquanto a externa está a um potencial $V_{1}$. As placas têm carga $Q$ de sinais opostos.

A fim de calcularmos a capacitância, primeiramente, escrevemos a Lei de Gauss,

$$
\oint \vec{E} \cdot d \vec{A}=\frac{Q_{i n t}}{\varepsilon_{0}}
$$

onde $\vec{E}$ é o campo elétrico, $\varepsilon_{0}$ é a permissividade elétrica do vácuo, $Q_{\text {int }}$ é a carga contida dentro de uma superfície gaussiana e a integral é calculada sobre esta.

Para o capacitor cilíndrico, as superfícies equipotenciais, na Fig. 1, são cilindros concêntricos, onde as linhas de força são retas radiais. Então, como superfície gaussiana, escolhemos um cilindro de comprimento $L$ e raio $r$. A equação (2) nos dá

$$
Q=\varepsilon_{0} E A=\varepsilon_{0} E(2 \pi r L)
$$

e, resolvendo para $E$, obtemos:

$$
E=\frac{Q}{2 \pi \varepsilon_{0} r L}
$$

que é o valor absoluto do campo elétrico radial.

Mas sabemos que

$$
\vec{E}=-\vec{\nabla} V
$$

onde $V$ é o potencial elétrico. Desta relação temos,

$$
V_{1}=\frac{Q}{2 \pi \varepsilon_{0} L} \ln \frac{R_{1}}{R_{2}}
$$

Mas, como desenvolvido em [1], a expressão do potencial elétrico em qualquer seção transversal entre as placas é dado por:

$$
V=\frac{V_{1}}{\ln \frac{R_{1}}{R_{2}}} \ln \frac{r}{R_{2}}
$$

Substituindo a relação (6) em (1), obtemos

$$
C=2 \pi \varepsilon_{0} \frac{L}{\ln \frac{R_{1}}{R_{2}}}
$$

Admitindo que as placas são homogêneas ao longo do comprimento do capacitor, projetamos o capacitor no plano definindo um $C^{\prime}$, denominado capacitância por unidade de comprimento, dado por:

$$
C^{\prime}=\frac{2 \pi \varepsilon_{0}}{\ln \frac{R_{1}}{R_{2}}}
$$

A partir dessa equação, vemos que a capacitância por unidade de comprimento de um capacitor cilíndrico concêntrico depende somente de fatores geométricos, $R_{1}$ e $R_{2}$.

\section{I.4 Capacitância com um Dielétrico}

Quando o espaço entre as placas de um capacitor está completamente preenchido com um material dielétrico, a capacitância fica aumentada por um fator $k$, chamado constante dielétrica, que é característica do material. Numa região completamente preenchida por um dielétrico, todas as equações eletrostáticas contendo $\varepsilon_{0}$ devem ser modificadas substituindo-se $\varepsilon_{0}$ por $k \varepsilon_{0}$.

Os efeitos da adição de um dielétrico ao capacitor podem ser entendidos fisicamente em termos da ação de um campo elétrico sobre dipolos elétricos permanentes ou induzidos na lâmina dielétrica. O resultado é a formação de cargas superficiais induzidas cuja presença resulta no enfraquecimento do campo no interior do dielétrico.

\section{Transformação Conforme}

O potencial elétrico no interior de um capacitor cilíndrico concêntrico é dado pela expressão (7), porém o nosso interesse é encontrar uma expressão do potencial elétrico para um capacitor cilíndrico para qualquer valor de excentricidade. Para isto, procuramos uma transformação conforme (TC) que leve a geometria nãoconcêntrica a uma onde os círculos estão concêntricos, para então escrever uma relação para o potencial semelhante à da equação (7), porém com dependência na excentricidade. A partir daí, questionamos qual é o comportamento da capacitância em função da excentricidade. $\mathrm{O}$ estudante que não estiver familiarizado com a técnica da TC pode encontrar no livro do Churchill [2] e do Arfken [3] uma boa leitura.

De acordo com [4 e 5], existe uma transformação conforme que leva a geometria de dois círculos não concêntricos (PLANO Z) a uma onde eles estão concêntricos (PLANO W), dada por:

$$
W=\frac{a Z+b}{b Z+a}
$$




$$
\bar{W}=\frac{a \bar{Z}+b}{b \bar{Z}+a}
$$

cuja inversa é

$$
Z=\frac{a W-b}{-b W+a}
$$

onde $a$ e $b$ são parâmetros que dependem da configuração dos círculos e as barras significam complexos conjugados. O leitor pode verificar como os pontos do Plano Z são mapeados no Plano $\mathrm{W}$, simplesmente colocando as coordenadas $(x, y)$ em $\mathrm{Z}$ e obter as correspondentes coordenadas $(u, v)$ de $\mathrm{W}$ (veja Apêndice para verificar como $(x, y)$ se relacionam com $(u, v))$. Na figura 2 ilustramos a transformação.

A condição para que exista a TC e a sua inversa, para todos pontos do plano, é que $\left(a^{2}-b^{2}\right) \neq 0$. Se $a^{2}=b^{2}$, a tranformação (10) torna-se uma constante. A escolha de que o círculo externo tenha raio unitário determina que $Z \bar{Z}=1$ (equação do círculo centrado na origem em variáveis complexas) e isto implica que $W \bar{W}=1$ para este círculo, logo $r_{1}=R_{1}=1$. Antes da TC, o círculo interno é dado pela equação,

$$
(Z+\delta)(\bar{Z}+\delta)=r_{2}^{2}
$$

e depois da TC, a equação para o círculo interno é

$$
W \bar{W}=r^{2}
$$

Conseqüentenente, com essas condições e após extensas manipulações algébricas, chegamos a:

$$
\begin{aligned}
r^{\prime 2} & =\frac{1}{2 r_{2}^{2}}\left\{\left[\left(1-2 \delta^{2}\right)+\left(\delta^{2}-r_{2}^{2}\right)^{2}\right]-\sqrt{\left[\left(1-2 \delta^{2}\right)+\left(\delta^{2}-r_{2}^{2}\right)^{2}\right]^{2}-4 r_{2}^{4}}\right\} \\
a^{2} & =\frac{1+\left[r^{\prime 2}\left(\delta^{2}-r_{2}^{2}\right)\right]}{\left(1+r_{2}^{2}-\delta^{2}\right)\left(1-r^{\prime 2}\right)} \\
b^{2} & =\frac{\left(r^{\prime 2}+\delta^{2}-r_{2}^{2}\right)}{\left(1+r_{2}^{2}-\delta^{2}\right)\left(1-r^{\prime 2}\right)}
\end{aligned}
$$

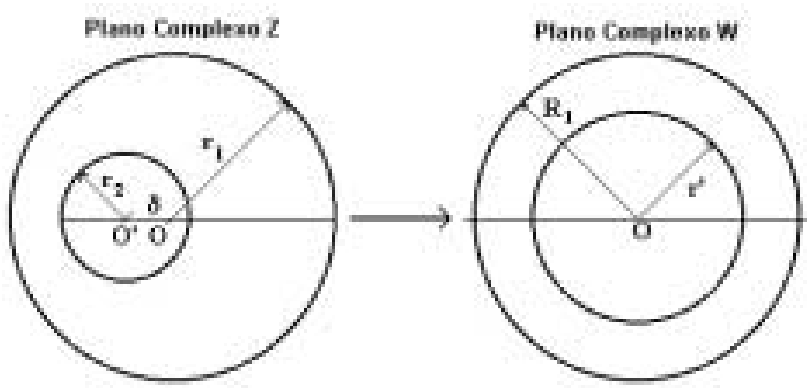

Figura 2. Ilustração esquemática de uma transformação conforme que leva dois círculos excêntricos a dois concêntricos. O raio interno $r_{2}$ é transformado em $r^{\prime}$ enquanto o raio externo $r_{1}$ é transformado para $R_{1}$.

estabelecendo uma dependência explícita de $r^{\prime}$ em termos dos parâmetros $\left(r_{2}, \delta\right)$. Note-se que a igualdade $\delta=0$ implica em $r^{\prime}=r_{2}, a=1, \quad b=0 \quad$ e a TC se torna a transformação identidade $Z=W$.

Uma vez que os principais passos da TC estão apresentados, a capacitância por unidade de comprimento para o capacitor cilíndrico excêntrico pode então ser calculada. Isto é, quando aplicamos a T.C, o raio interno passa assumir o valor $r^{\prime}$, e os cilíndros ficam concêntricos. Nesta configuração, a capacitância é dada pela equação (9) e é reescrita como:

$$
C^{\prime}=\frac{2 \pi \varepsilon_{0}}{\ln \frac{1}{r^{\prime}}}
$$

mostrando que $C^{\prime}=f\left(r^{\prime}\left(r_{2}, \delta\right)\right)$ e que depende portanto, implicitamente, da excentricidade $\delta$.

Para que o leitor possa ter um pouco mais de contato com a álgebra complexa da TC, apresentamos um apêndice na Seção IV, relacionando as variáveis do plano complexo $\mathrm{W}$, que representa a geometria concêntrica, com as variáveis do plano complexo Z, que representa a geometria não-concêntrica.

\section{Resultados e Conclusões}

Com relação à equação (18), nós observamos que, para todo $r^{\prime}<1$, se $r^{\prime}$ decresce, a capacitância decresce. O contrário também se verifica: se $r^{\prime}$ cresce, a capacitância aumenta. Para o limite $r^{\prime}=1$, que representa o caso quando as placas estão em contato, a equação (18) não está definida pois $C^{\prime}$ tende ao infinito.

Embora seja conhecido que a capacitância sempre cresce com a aproximação de condutores de cargas opostas, neste sistema à medida que o círculo interno se aproxima do círculo externo pela esquerda, com a variação da excentricidade $\delta$, ele se afasta do círculo externo pela direita. Isso torna interessante o estudo da capacitância deste sistema.

Um ponto importante é entender a relação entre $r^{\prime}$ e os parâmetros $\left(r_{2}, \delta\right)$. A Fig. 3 mostra um gráfico de $r^{\prime}$ (raio interno transformado) contra $r_{2}$ (raio original do círculo excêntrico) para um $\delta$ não nulo fixado, 
onde observamos que $r^{\prime}$ é sempre maior que $r_{2}$. Nós usamos vários valores para $\delta$ para checar este resultado e observamos para qualquer $\delta, \quad r^{\prime}>r_{2}$.

A excentricidade contribui na equação (18) fornecendo um valor de $r^{\prime}$ maior do que $r_{2}$, e de acordo com a análise anterior a capacitância por unidade de comprimento será maior que o caso concêntrico correspondente, o qual contribui com $r_{2}$ na equação (18). A Fig. 4 mostra a capacitância como função do raio interno para um capacitor concêntrico e excêntrico.

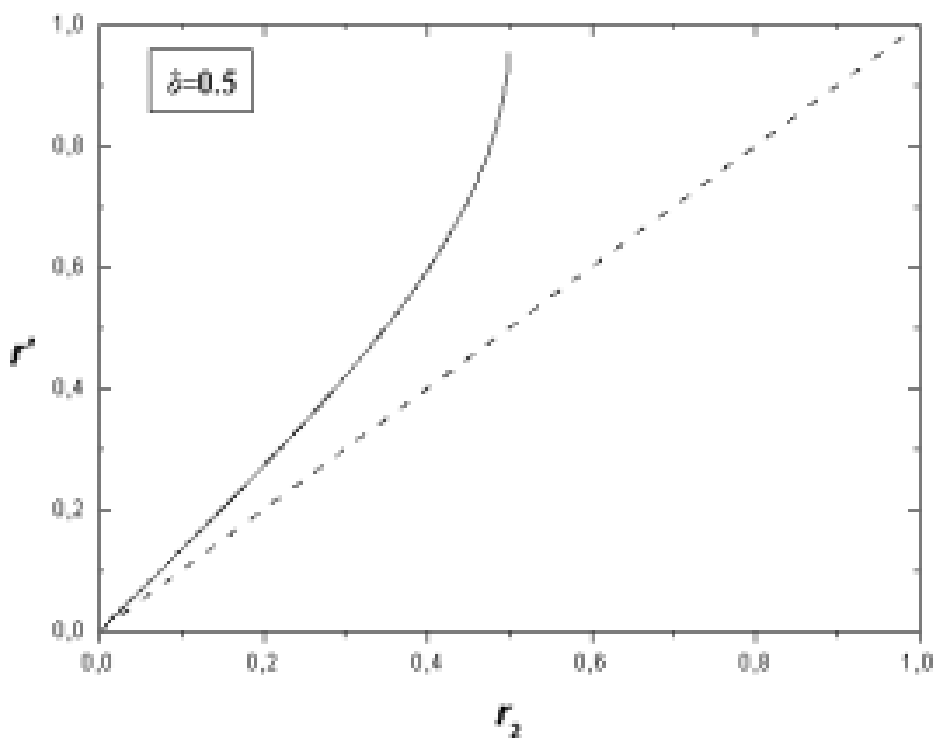

Figura 3. Gráfico da equação (15), $r^{\prime}$ como função de $r_{2}$ com o valor de $\delta=0.5$ fixado. Observamos que $r^{\prime}$ é sempre maior que $r_{2}$. A reta de $45^{\circ}$ é para facilitar a visualização.

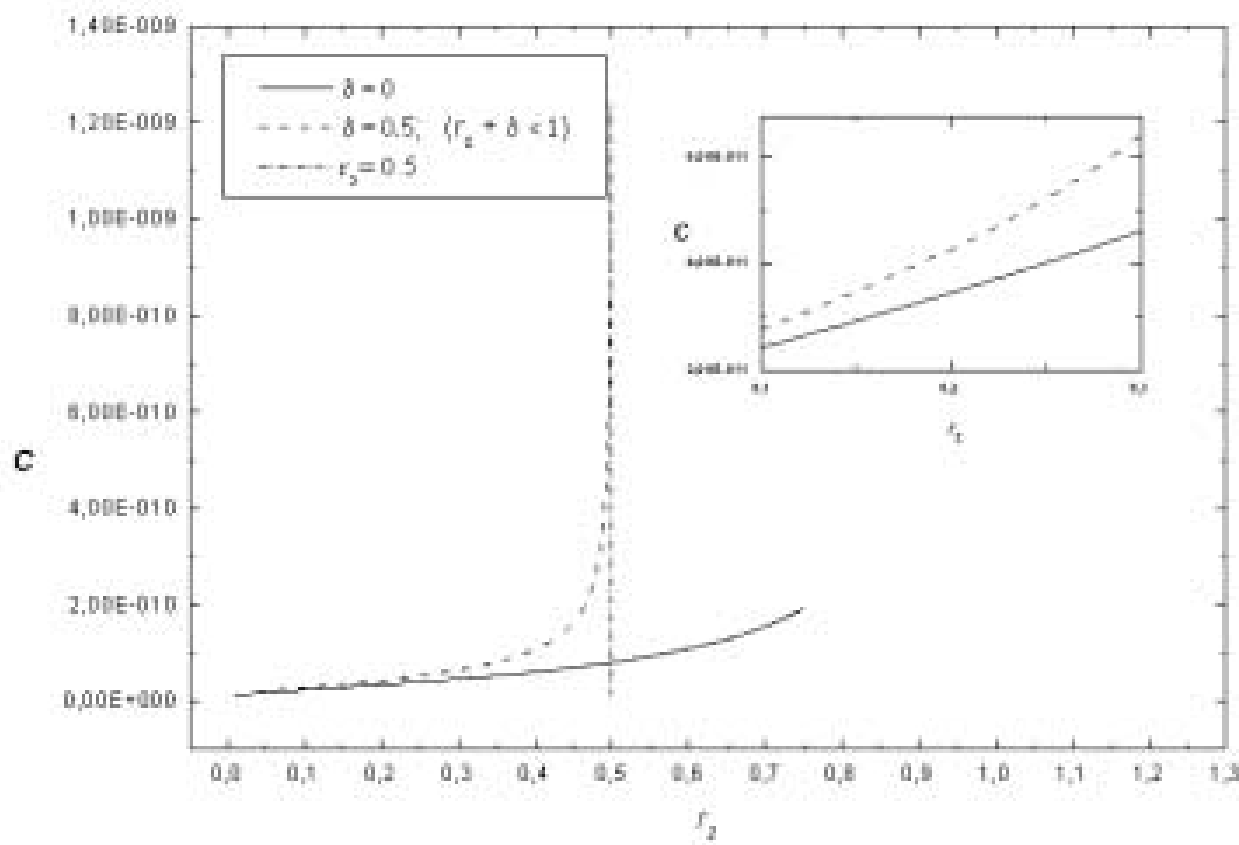

Figura 4. A capacitância por unidade de comprimento como função de $r_{2}$ para as duas configurações, a concêntrica e a excêntrica. Para cada valor de $r_{2}$, o excêntrico contribui com $r^{\prime}$ na equação (20). 
Conseqüentemente, é possível melhorar a performance do capacitor sem propriamente fazer uma mudança geométrica; as placas continuam sendo cilíndricas, mas dispostas de maneira diferente. Este resultado pode ser checado experimentalmente e pode ser de interesse para o desenvolvimento de componentes eletrônicos. Os cálculos aqui apresentados servem de suporte teórico para guiar uma montagem experimental a fim de medir a capacitância de um capacitor cilíndrico excêntrico.

Para concluir, queremos enfatizar que os nossos resultados permitem economizar material na construção de um capacitor cilíndrico. Suponha-se que o objetivo seja obter um capacitor com uma determinada capacitância $C$, por exemplo. Isso pode ser alcançado por dois caminhos, um com a geometria concêntrica para um dado raio interno $R_{2}$, e outro com a geometria excêntrica para um raio interno $r_{2}<R_{2}$, o qual, quando teoricamente transformado, produzirá o $R_{2}$ desejado e a mesma capacitância $C$. Ou seja, a capacitância pode ser amplificada e a quantidade de material para construir as placas do capacitor pode ser reduzida com a geometria excêntrica.

\section{Apêndice}

A seguir, fazemos um quadro indicando as variáveis nas duas geometrias: caso concêntrico e excêntrico.

$\begin{array}{cc}\text { PLANO W } & \text { PLANO Z } \\ \text { (Concêntrico) } & \text { (Excêntrico) } \\ W=u+i v & Z=x+i y \\ u, v & x, y \\ r, \theta & \rho, \varphi \\ u=r \cos \theta & x=\rho \cos \varphi \\ v=r \sin \theta & y=\rho \sin \varphi\end{array}$

Tabela 1. Variáveis referentes ao PLANO W e PLANO Z.

A partir desta tabela, fazemos conexões entre as variáveis do plano concêntrico (PLANO W) e excêntrico (PLANO Z).

Escrevemos as variáveis polares do PLANO W (Concêntrico) em função das variáveis polares do PLANO Z. Para isto, utilizamos a relação (10),

$$
W=\frac{a Z+b}{b Z+a}
$$

Mas como $Z=x+i y \quad$ e $\quad W=u+i v \quad$ então obtemos,

$$
W=(u+i v)=\frac{a(x+i y)+b}{b(x+i y)+a}
$$

o que nos dá,

$$
\begin{aligned}
u & =\frac{a b\left(x^{2}+y^{2}\right)+a b+\left(a^{2}+b^{2}\right) x}{b^{2}\left(x^{2}+y^{2}\right)+2 a b x+a^{2}} \\
v & =\frac{\left(a^{2}-b^{2}\right) y}{b^{2}\left(x^{2}+y^{2}\right)+2 a b x+a^{2}}
\end{aligned}
$$

Substituindo as relações $x=\rho \cos \varphi$ e $y=\rho \sin \varphi$ nas relações (20) e (21) acima, obtemos:

$$
\begin{aligned}
u & =\frac{a b \rho^{2}+a b+\left(a^{2}+b^{2}\right) \rho \cos \varphi}{b^{2} \rho^{2}+2 a b \rho \cos \varphi+a^{2}} \\
v & =\frac{\left(a^{2}-b^{2}\right) \rho \sin \varphi}{b^{2} \rho^{2}+2 a b \rho \cos \varphi+a^{2}}
\end{aligned}
$$

Sabendo que $r^{2}=u^{2}+v^{2}$ e $\tan \theta=\frac{v}{u}$, chegamos às expressões seguintes:

$$
\begin{gathered}
r^{2}=\frac{a^{2} \rho^{2}+2 a b \rho \cos \varphi+b^{2}}{b^{2} \rho^{2}+2 a b \rho \cos \varphi+a^{2}} \\
\theta=\tan ^{-1}\left(\frac{\rho \sin \varphi\left(a^{2}-b^{2}\right)}{a b\left(\rho^{2}+1\right)+\rho \cos \varphi\left(a^{2}+b^{2}\right)}\right)
\end{gathered}
$$

que determinam as relações que transformam as variáveis polares do PLANO Z não-concêntrico com as do PLANO concêntrico W.

Por outro lado, uma vez que existe a TC inversa, podemos obter as variáveis do PLANO $\mathrm{Z}$ em termos daquelas do PLANO W. As equações são análogas às equações (22), (23), (24) e (25).

Utilizamos agora a equação (12):

$$
Z=\frac{a W-b}{-b W+a}
$$

equação (12) fica:

$$
\text { Com } W=u+i v \text { e } Z=x+i y \text { a }
$$

$$
Z=(x+i y)=\frac{a(u+i v)-b}{-b(u+i v)+a}
$$

o que nos dá:

$$
\begin{aligned}
x & =\frac{-a b\left(u^{2}+v^{2}\right)-a b+\left(a^{2}+b^{2}\right) u}{b^{2}\left(u^{2}+v^{2}\right)-2 a b u+a^{2}} \\
y & =\frac{\left(a^{2}-b^{2}\right) v}{b^{2}\left(u^{2}+v^{2}\right)-2 a b u+a^{2}}
\end{aligned}
$$

Substituindo as relações $u=r \cos \theta$ e $v=r \sin \theta$ nas relações (27) e (28) acima obtemos:

$$
\begin{aligned}
x & =\frac{-a b r^{2}-a b+\left(a^{2}+b^{2}\right) r \cos \theta}{b^{2} r^{2}-2 a b r \cos \theta+a^{2}} \\
y & =\frac{\left(a^{2}-b^{2}\right) r \sin \theta}{b^{2} r^{2}-2 a b r \cos \theta+a^{2}}
\end{aligned}
$$


Sabendo que $\rho^{2}=x^{2}+y^{2}$ e $\tan \varphi=\frac{y}{x}$, chegamos às seguintes relações inversas:

$$
\begin{gathered}
\rho^{2}=\frac{a^{2} r^{2}-2 a b r \cos \theta+b^{2}}{b^{2} r^{2}-2 a b r \cos \theta+a^{2}} \\
\varphi=\tan ^{-1}\left(\frac{r \sin \theta\left(a^{2}-b^{2}\right)}{r \cos \theta\left(a^{2}+b^{2}\right)-a b\left(r^{2}+1\right)}\right)
\end{gathered}
$$

que transformam as variáveis polares do PLANO Z (Não-Concêntrico) e o PLANO W (Concêntrico).

\section{Agradecimentos}

Agradecemos ao apoio financeiro da Fapesp na concessão da Bolsa de Iniciação Científica identificada pelo Processo 00/01304-3.

\section{Referências}

[1] P. Lorrain, D.R. Corson Eletromagnetic Fields and Waves $2^{a}$ ed.(197 0), W.H. Freeman and Company NY.

[2] R.V. Churchill, Variáveis Complexas e suas Aplicações, (1975) McGraw-Hill, SP.

[3] G.B. Arfken, H.J. Weber, Mathematical Methods for Physicists $4^{a}$ ed. (1995), Academic Press, NY.

[4] O. Bohigas, D. Boosé, R. Egydio de Carvalho, V. Marvulle, Nucl. Phys A560(1993) 197.

[5] H. Kober Dictionary of Conformal Representations, p.30, NY: Dever Publications Inc. (1957). 